\title{
Cervical cytology after 2000: where to go?
}

\author{
C J L M Meijer, J M M Walboomers
}

Since the introduction of the Pap smear by George Papanicolaou, cervical cytology has become the main diagnostic tool to detect cervical cancer. Nowadays cervical cytology is not only used for the detection of overt cancer cells, but also for finding precursor lesions of cervical cancer - that is, cervical intraepithelial lesions grades I-III (CIN). The success of cervical cytology has often been demonstrated by the decrease in the prevalence of cervical cancer in countries where population based organised cervical cancer screening programmes have been introduced. ${ }^{1}$ However, it should be realised that the use of the Pap smear to detect cervical cancer and its precursor lesions is in fact also its tragedy, because the smears read as false negative are often precursor lesions.

Principally, cervical cytology can be used in the following ways:

- for the detection of cervical cancer and its precursor lesions, either in indicative cervical smears or in population based screening programmes;

- for the management of women with abnormal cytology; and

- to detect residual or recurrent cervical lesions after treatment.

The limited sensitivity and specificity of cervical cytology for detecting cervical cancer and its precursor lesions has been the subject of considerable debate in the last decades. To overcome these problems, several measures have already been taken to improve the end results of smear reading. These include the use of brushes instead of wooden spatulas, optimisation of staining techniques, better training of technicians, and rescreening of smears.

These measures have resulted in some improvement in the quality of reading cervical smears. However, it has now become clear that even in high quality laboratories with carefully controlled cervical smear reading, the presence of false positive and false negative smear results cannot entirely be prevented.

Given these limitations of cervical cytology the question is whether we can improve the reading of cervical smears. The use of two new technologies may achieve such improvement:

1. The development of liquid based cytology preparations;

Department of Pathology, Academic Hospital Vrije Universiteit Amsterdam, The Netherlands C J L M Meijer J M M Walboomers

Correspondence to: Prof Dr Meijer email: cjlm.meijer@azvu.nl preparing thin layers of cells by means of a special device, much better cytological preparations are obtained, lacking damaged and dead cells and showing mostly isolated cells. This leads to quicker and better smear reading (Cytec ${ }^{\circledR}$, Autocyte ${ }^{\circledR}$ ). Because not all the cells are used for the preparation of the smear, additional techniques such as HPV detection, immunohistochemical staining for cell cycle indices, tumour suppressor genes or oncogenes, and additional molecular techniques can be used, making this technique very important. As diagnostic cell groups are absent in thin layer preparations, a point of discussion is the question of how representative the smear prepared from liquid cytology is, compared with the original smear. It has been concluded that differences between a classical Pap smear and cell preparations obtained by liquid cytology are too small to influence Pap classification significantly, thus suggesting that liquid based cytology preparation is the technique to be used in the future. ${ }^{2}$ A disadvantage of the technique is that it is still too expensive for general use and until a significant decrease in price has been announced, the introduction of this technique into the routine setting will be considerably delayed. ${ }^{3}$

\section{Automated screening of cervical smears}

Significant contributions were made by the Papnet system, whereby with the aid of neural network technology 128 areas from the most abnormal cells in a smear were digitised so that the operative can focus on these areas to obtain a rapid and reliable impression of the degree of cellular abnormality in the smear. Evaluations of the system were positive. ${ }^{4}$ However, that particular system will not be supported commercially in the future, and the technology has been transferred to another company, Neopath. This company has now brought out the Autopap 300 QC system. This system, also based on neural network technology, divides the smears into five classes, varying from very suspicious of cervical cancer to normal. Depending of the sensitivity of the system and the population screened, between $25 \%$ and $40 \%$ of the smears in a population based screening programme can be passed without further screening. Neural network technology is still advancing and obviously automated cervical cytology screening based on this technology will have a place in the future.

Again, an important problem here is the price. As the costs of cervical smears are heavily controlled by health insurance companies or government institutions, the price offered at the moment is too high to implement these systems in population based screening programmes. When the costs of such systems are not adapted to the available resources, the effect will be that their implementation will result in less screening opportunities for high risk women, without a net positive effect on the 
quality of cervical cancer screening. One way to overcome these problems is that screening should be performed in laboratories where at least 200000 smears are read. For economic reasons and in order to maintain standards, it is impossible to implement these systems in smaller laboratories.

\section{High risk HPV testing}

Another way ahead is the use of human papillomavirus (HPV) testing. Recent investigations clearly show that persistent infection with one of the 15 high risk HPV types is necessary for the development and maintenance of CIN lesions and the subsequent progression to cervical cancer. ${ }^{5}{ }^{6}$ Prospective clinical follow up studies of women with normal and abnormal cervical smears clearly show that high risk HPV infection precedes the development of CIN III (the most advanced precursor lesion of cervical cancer). ${ }^{578}$ Walboomers et al were one of the first to show that, in women with cervical cancer, high risk HPV was always present in the preceding abnormal smears and was of the same high risk HPV type as that present in the carcinoma. High risk HPV was even present in normal smears taken up to 17 years before the development of the carcinoma ( ${ }^{9}$ and Jacobs MV et al, submitted for publication). These studies have resulted in the following model: when women start sexual intercourse, an infection with high risk HPV is acquired. About $80 \%$ of the women clear this infection without developing cervical lesions. The rest $(20 \%)$ of the women develop CIN lesions. Most of these women with abnormal smears (about 80\%) show spontaneous regression of the cervical lesion and obtain normal cytology when high risk HPV is cleared. Those women who cannot clear the virus and have a persistent high risk HPV infection will develop invasive cervical cancer, but additional genetic changes, such as activation of cellular oncogenes or loss of tumour suppressor genes, are necessary for this progression from CIN III to invasive carcinoma. Thus the detection of high risk HPV in cervical smears is a powerful tool for detecting those at risk of developing cervical cancer. ${ }^{10}$

\section{Population based cervical cancer screening}

Several strategies are now proposed to optimise the detection of cervical cancer and its precursor lesions. ${ }^{11}$ One is the combination of cervical cytology with high risk HPV testing. In this approach, women with abnormal smears are only sent to a gynaecologist when high risk $\mathrm{HPV}$ is present. Women with normal cervical smears but with high risk HPV in their smears are followed up for a year and retested. This strategy enables us to increase the screening interval between two successive rounds of screening. Women who have high risk HPV negative normal smears should only be rescreened after 8-10 years, on the basis that the mean time necessary for the development of cervical cancer from a smear with mild dyskaryosis is 13.1 years. ${ }^{12}$ Moreover, by this approach overtreatment is prevented, as a sub- stantial number of women with abnormal smears and high risk HPV negative lesions are managed using a wait-and-see policy.

Another approach that can be expected in the future is to first screen women for the presence of high risk HPV and then test only those who are high risk HPV positive for the presence of abnormal cells in the Pap smear. This approach is straightforward and leads to an enormous cost reduction.

At present, clinicians are reluctant to follow this strategy, because CIN III lesions are occasionally found to be negative for high risk HPV types and as their clinical course is unkown lawsuits are expected. However, when further evaluations of large, population based studies are published, in which the performance of high risk HPV testing and classical cytology are evaluated, this strategy will certainly be implemented. It seems logical to assume that in the future these HPV positive Pap smears will then be evaluated by automated screening systems as discussed above. ${ }^{13}$ This approach is very important for developing countries in which high quality cervical cytology laboratories are difficult to organise. Implementation of one screening round between 30 and 35 years of age in these developing countries, using liquid based high risk HPV testing followed by automated screening of thin layer preparations from women who tested high risk HPV positive, will select women for colposcopically directed biopsy and treatment. This approach could reduce the prevalence of cervical cancer in these countries by about $70 \%$, based on the fact that high risk HPV testing can detect almost all CIN III lesions and cervical cancers. Moreover, this age (30-35 years) is more than 10 years after the peak prevalence of HPV infection in these countries, and $70 \%$ of all cervical cancers have developed by that age.

\section{Management of women with abnormal cytology}

A Pap smear showing mild dysplasia or atypical squamous cells of undetermined significance (ASCUS) is a problem for management as only $5-10 \%$ of women with such smear results harbour serious disease. ${ }^{14}$ High risk HPV testing helps to identify the women who have underlying CIN II or CIN III and can be referred for colposcopy directed biopsy, based on a single screening round of cytology and high risk HPV testing. An even more advanced approach was suggested by Nobbenhuis et al, who showed that in women with mild to moderate dysplasia only those who have a repeated high risk HPV positive Pap smear after six months should be referred for colposcopy, thereby preventing overtreatment of non-progressive CIN lesions. ${ }^{5}$ From these observations it can be concluded that the future of cervical cytology is closely linked to high risk HPV testing.

\section{Detection of residual or recurrent CIN lesions after treatment}

After treatment of CIN lesions by diathermic ablation or exconisation, $10-15 \%$ of the women have residual or recurrent lesions after one year. ${ }^{15}{ }^{16}$ Cervical cytology is often the first 
test used to detect such lesions at follow up. These women need colposcopically directed biopsy and treatment by therapeutic exconisation of the cervix. Recent reports show that a cervical smear is not a good discriminator of the presence or absence of a CIN lesion after treatment. ${ }^{17}$

Recent studies reveal that high risk HPV is present in $96-100 \%$ of the recurrent CIN lesions. ${ }^{18}$ Preliminary studies indicate that high risk HPV testing is a much better predictor of recurrent CIN lesion than a cervical smear (Nobbenhuis MAE, et al; submitted for publication). We are presently studying, in a randomised trial, the effect of treatment of CIN lesions on the basis of either cytology or high risk HPV testing. Thus this is another area where cervical cytology meets high risk HPV testing.

\section{Where do we go now?}

The ultimate way forward will be the use of liquid based cytology preparations in combination with HPV testing and automated screening. The thin layer preparations are read by automated screening devices and high risk HPV testing can be done directly from the same liquid preparation of cervical cells. Moreover, in this approach only high risk HPV positive cytomorphologically normal smears would be rescreened for the presence of abnormal cells, as in $5-7 \%$ of the high risk HPV positive, cytomorphologically normal smears abnormal cells are still found. ${ }^{10}$ This will certainly reduce the number of false positive smears. Although in the next 5-10 years cervical cytology will be done in conjunction with high risk HPV testing, the results of large population based studies will finally lead to high risk HPV testing in the first place, followed by cytological screening of the HPV positive smears using automated systems. As the costs of screening for cervical cancer are tightly controlled by health insurance companies and governmental regulations, it will be difficult to implement these new technologies. At this moment the introduction of such techniques will certainly require an increase in budgets, but this will decrease after 5-10 years. It is the responsibility of the health insurance companies, government, pathologists, and gynaecologists to implement these new techniques for screening in cervical cancer at a rea- sonable price, because then we can go for the ultimate goal-the prevention of death from cervical cancer.

We would like to thank Drs E Risse and J Lindeman for fruitful discussion and advice. Tonia Borgmann is thanked for carefully typing the manuscript, as always.

1 Hakema M, Louhivuori K. A screening program for cervical cancer that worked. Cancer Surv 1988;7:403-16.

2 Carpenter AB, Davey DD. Thin Prep Pap test: performance and biopsy follow-up in a university hospital. Cancer 1999; 87:105-12.

3 Sauvageau GF, Grimes DA. New technologies in cervical cytology screening: a word of caution. Obstet Gynecol 1999;94:307-10.

4 Bosanquet N, Coleman DV, Dore CJ, et al. Assessment of automated primary screening on PAPNET of cervical smears in the Prismatic trial. Lancet 1999;353:1381-5.

5 Nobbenhuis MAE, Walboomers JMM, Helmerhorst TJM, et al. Relation of human papillomavirus status to cervical lesions and consequences for cervical cancer screening: a prospective study. Lancet 1999;354:20-5.

6 Walboomers JMM, Jacobs MV, Manos MM, et al. Human papillomavirus is a necessary cause of invasive cervical cancer worldwide. F Pathol 1999;189:12-19.

7 Rozendaal L, Walboomers JMM, van der Linden JC, et al. PCR-based high risk HPV test in cervical cancer screening gives objective risk assessment of women with cytomorphologically normal cervical smears. Int f Cancer 1996;68:7669.

8 Remmink AJ, Walboomers JMM, Helmerhorst TJM, et al. The presence of persistent high risk HPV human papillomavirus genotypes in dysplastic cervical lesions is papsillomavirus genotypes in dysplastic cervical lesions is 36 months. Int f Cancer 1995;61:1-6.

9 Walboomers JMM, de Roda Husman AM, Snijders PJF, et al. Human papillomavirus in false negative smears: implications for screening for cervical cancer. $\mathcal{F}$ Clin Pathol 1999;48:498-503.

10 Meijer CJLM, Rozendaal L, Van der Linden JC, et al. Human papillomavirus testing for primary cervical cancer screening. In: Franco E, Monsenego J, eds. New developments in cervical cancer screening and prevention. Oxford: Blackwell Science, 1997:338-47.

11 Meijer CJLM, van den Brule AJC, Snijders PJF, et al. Detection of human papillomavirus in cervical scrapes by the polymerase chain reaction in relation to cytology: possible implications for cervical cancer screening. In: Epidemiology of cervical cancer and human papillomavirus.
Lyon: IARC, 1992:271-81.

12 van Ballegooijen $M$, Bos $A B$, van Marle $M E$, et al. A first description of the screening practice in the Netherlands in 1994 based on data from Palga. Rotterdam: Erasmus University, 1997. [In Dutch.]

13 Leiman G. Thin layer cytology meets viral hybrid capture. Adv Anat Pathol 1999;6:161-4.

14 Manos MM, Kinney WK, Hurley LB, et al. Identifying women with cervical neoplasia. Using human papillomavirus DNA testing for equivocal Papanicolaou results. $\mathscr{f} A M A$ 1999;281:1605-10.

15 Bistoletti P, Zellbi A, Moreno-Lopez J, et al. Genital papillomavirus infection after treatment for cervical intraepithelial neoplasia (CIN) III. Cancer 1988;62:2056-9.

16 Elfgren K, Bistoletti P, Dillner L, et al. Conization for cervi$\mathrm{cal}$ intraepithelial neoplasia is followed by disappearance of human papillomavirus deoxyribonucleic acid and a decline human papillomavirus deoxyribonucleic acid and a decline papillomavirus antigens. Am f Obstet Gynecol 1996;174: papillomavir

17 Bollen LJM, et al. Prediction of recurrent cervical dysplasia by human papillomavirus detection among patients with by human papillomavirus detection among patients
abnormal cytology. Gynecol Oncol 1999;72:199-201.

18 Chua KL, Hjerpe A. Human papillomavirus analysis as a prognostic marker following conization of the cervix uteri. Gynecol Oncol 1997;66:108-13. 